\title{
ÉTUDE BIOMÉTRIQUE DES PLACENTOMES DANS LES GESTATIONS SIMPLES OU GÉMELLAIRES DES BOVINS ${ }^{(1)}$
}

\author{
J. TESTART et F. DU MESNIL DU BUISSON \\ Institut national de la Recherche agronomique \\ Station centrale de Physiologie animale, 78 -Jouy-en-Josas, France \\ Laboratoire de Physiologie de la Reproduction, 37 - Nouzilly, France
}

\section{INTRODUCTION}

Comment l'utérus de la Vache est-il capable de s'adapter à l'implantation de plusieurs foetus?

Le développement cotylédonnaire est certainement l'un des éléments les plus importants qui conditionnent la croissance des fotus. Nous savons par exemple que chez la Brebis, femelle dont la placentation est comparable à celle de la Vache, le débit sanguin au niveau cotylédonnaire représente durant les derniers mois de la gestation, 84 p. Ioo du débit utérin (Makowski et al., Ig68).

Or, le nombre de caroncules étant fixe pour un utérus donné, la part relative de chaque fœtus diminue normalement au fur et à mesure que le nombre des fœtus augmente.

Aussi, en cas de gémelliparité, les fœetus ne pourraient poursuivre un développement satisfaisant, si un mécanisme compensatoire n'intervenait soit dans le débit sanguin utérin, soit dans le taux de mobilisation des caroncules ou dans le développement cotylédonnaire.

Dans un premier temps, nous avons donc étudié le développement des cotylédons sous l'angle de leur nombre, de leur poids individuel et de leur répartition dans l'utérus au cours de gestations simples ou doubles.

\section{MATÉRIEL E'T MÉTHODES}

Des utérus gravides ont été collectés aux abattoirs de la Villette sans tenir compte de l'âge ni du la race de la vache $\left({ }^{2}\right)$. Ils ont été conservés à la chambre froide à $+4^{\circ} \mathrm{C}$ in toto et disséqués au laboratoire dans la semaine. Nous envisagerons ici seulement les mesures pondérales des cotylédons.

(1) La majeure partie de ce texte a déjà été publié dans les Annales de Biologie animale, Biochimie Biophysique, 6, 483-493 (I 966 ). mande.

(2) La majorité des vaches abattues à la Villette sont de race Française Frisonne Pie Noire ou Nor- 
L'ensemble des mesures a été rapporté au poids des fœetus puisque l'âge ne pouvait être connu que par estimation d'après les données de Winters et al. (1953) et Fournier (I963) (tabl. r).

Quatre-vingts utérus gravides ont été étudiés, dont 44 issus d'animaux en gestation simple et 36 issus d'animaux en gestation gémellaire.

TABLEAU I

\begin{tabular}{l|c|c|c|c|c|c|c}
\hline Poids du fœetus (g) & 30 & 100 & 300 & 1000 & 3000 & 6000 & 9000 \\
\hline $\begin{array}{c}\text { Age approximatif } \\
\text { (jours) } \ldots \ldots \ldots\end{array}$ & 65 & 80 & 100 & 125 & 150 & 185 & 205 \\
\hline
\end{tabular}

\section{RÉSULTATS ET DISCUSSION}

\section{Nombre de placentomes en fonction du moment de la gestation et du type de gestation}

a) Répartition des placentomes par corne.

Le nombre de caroncules de l'utérus bovin est assez variable. Une étude préalable sur 7o utérus non gravides indique une moyenne de 69 par corne utérine (de 90 à I 80 pour l'ensemble de l'utérus).

a) Gestation simple.

La transformation des caroncules en cotylédons maternels porte toujours, dans la corne gravide, sur un effectif supérieur à celui qui est affecté dans la corne non gravide (tabl. 2 A).

I. nombre de placentomes dans chacune des deux cornes peut varier considérablement selon les individus puisque dans la corne gravide il se situe entre 38 et Ioo $(\bar{m}=68)$ et dans la corne non gravide entre o et $70(\bar{m}=28)$. Pour $75 \mathrm{p}$. Ioo des vaches, il est compris entre 40 et 80 dans la corne gravide et entre o et 40 dans la corne non gravide.

L'effectif caronculaire intéressé qui se transforme en cotylédon semble augmenter jusqu'au $4^{\mathrm{e}}$ mois de gestation, puis se stabilise. Sur les utérus gestants de plus de 4 mois, nous avons dénombré en moyenne 73 cotylédons dans la corne gestante et 34 dans l'autre.

b) Gestation gémellaire.

La répartition est très différente suivant que la gestation gémellaire s'établit dans une corne ou dans les deux.

I. Si les 2 fœtus occupent la même corne utérine (tabl. 2 в), le nombre total des placentomes n'est pas plus élevé qu'en gestation unifœetale. La répartition entre les deux cornes diffère peu des gestations simples.

2. Si chaque corne utérine est occupée par I fœetus (tabl. 2 c) le nombre total de placentomes est beaucoup plus grand que dans les deux cas précédents (moyenne I28). Il est très proche du nombre total de caroncules disponibles (moyenne I38).

De plus, chaque corne participe pour $5^{\circ} \mathrm{p}$. Ioo à l'effectif total de placentomes alors que la corne non gravide des gestations unilatérales, simples ou gémellaires portait seulement $30 \mathrm{p}$. Ioo des placentomes. 
PLACENTOMES DES BOVINS

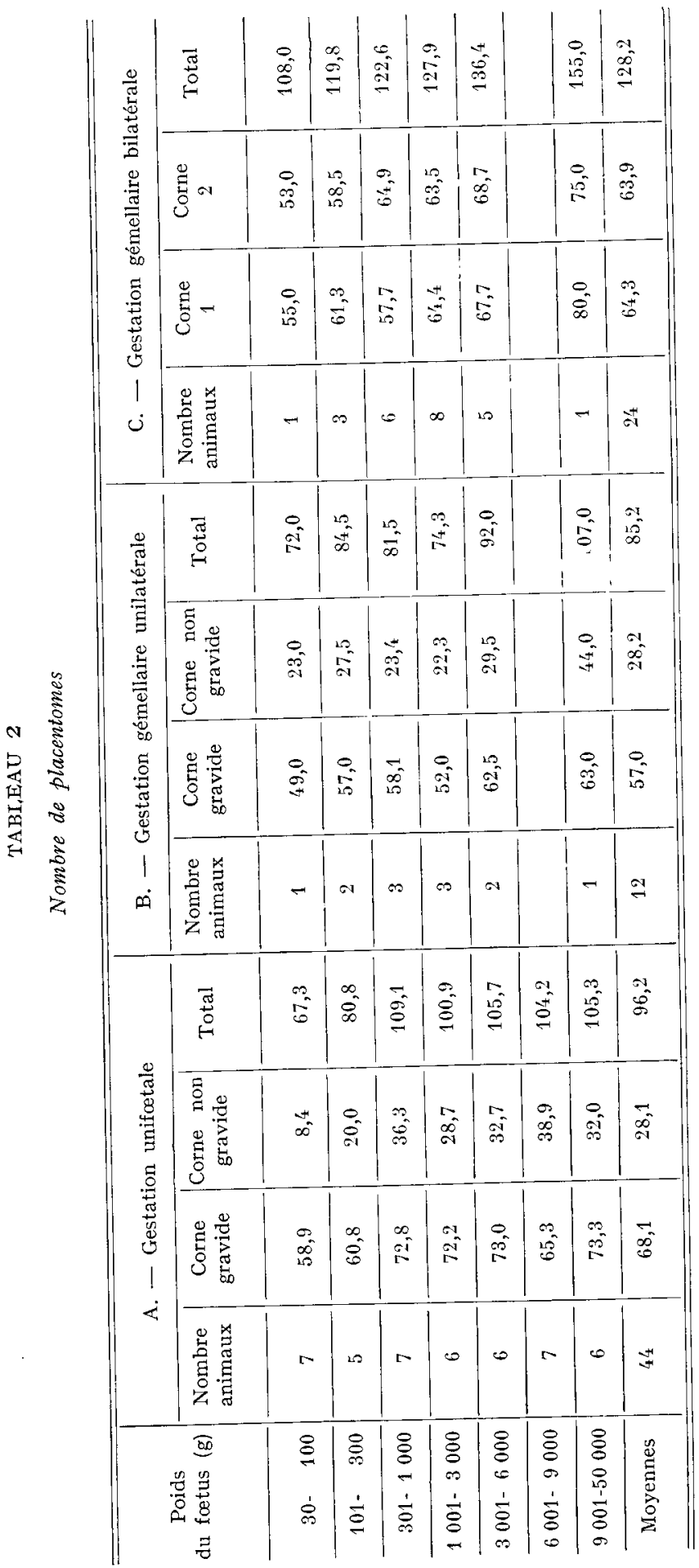


b) Répartition des placentomes le long des cornes.

On a divisé arbitrairement chaque corne utérine en 3 segments égaux : supérieur, moyen et inférieur et on a dénombré les placentomes dans chacun de ces segments (fig. I).

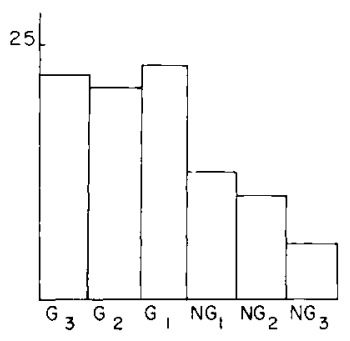

A. Gestation unifoetale

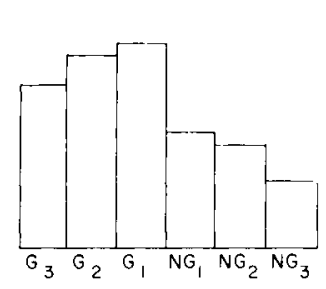

B. Gestation gemellaire unilatérale

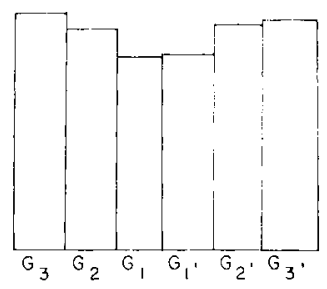

C Gestotion gemellcire bilaterole

FIG. r. -- Répartition des placentomes

$\mathrm{C}=$ corne gravide $\quad \mathrm{NG}=$ corne non gravide

I, 2, $3=$ tiers inférieur, moyen et supérieur des différentes cornes

Dans la ou les cornes gravides les trois segments montrent un nombre analogue de cotylédons.

$\mathrm{Au}$ contraire, dans la corne non gravide en gestation unilatérale, unifœetale ou gémellaire, la densité des placentomes décroit de la base (corps utérin) au sommet de la corne (jonction utéro-tubaire).

2. Poids individuel des placentomes en fonction du moment de la gestation, du type de gestation et de leur position dans l'utérus

Le poids des placentomes augmente pendant la gestation ; les cotylédons maternels et fœtaux sont de poids semblable jusque vers 4 mois, puis le cotylédon maternel devient plus lourd que le cotylédon fœetal dans tous les types de gestation. Le tableau 3 illustre cette croissance en ce qui concerne la gestation unifœetale.

a) Gestation unifoctale et gestation gémellaire bilatérale.

Les placentomes dans la ou les cornes gestantes semblent croître à la même allure dans ces deux cas (fig. 2).

Par exemple, au $5^{\mathrm{e}}$ mois de gestation (poids de fœetus : I 000 à 3 ooo g), le poids moyen d'un placentome du segment médian est de $20,2 \mathrm{~g}$ dans la corne gravide en gestation simple, de $23,5 \mathrm{~g}$ dans chacune des cornes gravides en gestation gémellaire bilatérale.

Les placentomes des segments de corne où se trouve le foetus (en général le médian) sont environ 2 fois plus lourds que les placentomes situés dans les segments adjacents. Le gradient qui s'établit à partir de la zone fœetale se poursuit progressivement vers la corne non gravide, dans le cas de gestation simple.

b) Gestation gémellaire unilatérale.

A partir du $\mathrm{IOo}^{\mathrm{e}}$ jour de la gestation, la vitesse de croissance des placentomes semble être à peu près le double de celle observée dans les deux cas précédents (fig. 2). 
PLACENTOMES DES BOVINS

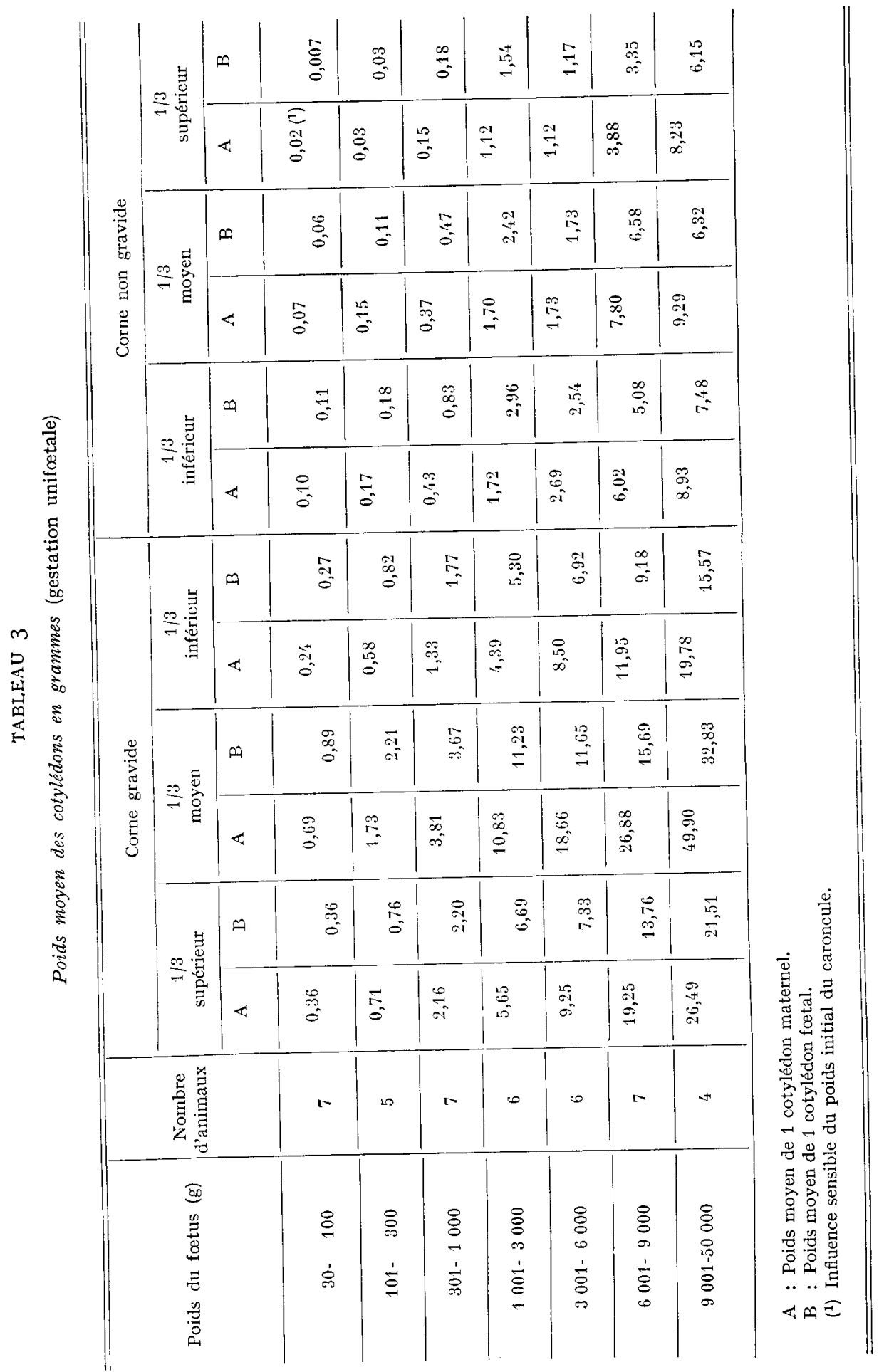


Cette augmentation de la vitesse de croissance atteint autant les placentomes situés dans la corne non gravide que dans l'autre. Un gradient comparable à celui qui a déjà été signalé s'établit à partir de l'insertion des fœetus, mais ceux-ci se trouvant tantôt l'un et l'autre dans le segment médian, tantôt chacun dans un des segments supérieurs, il apparaît dans cette série des différences entre vaches, quant à l'origine du gradient.

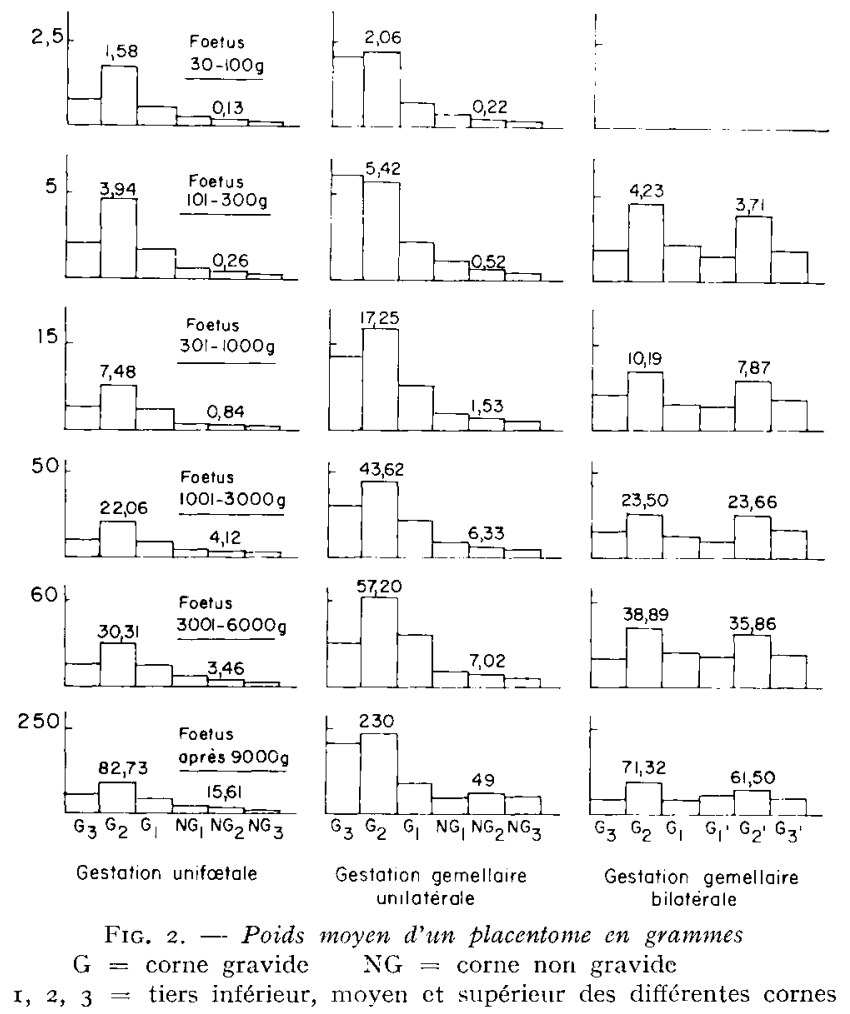

L'augmentation de la vitesse de croissance de placentomes aboutit, à la fin de la gestation gémellaire unilatérale, à la formation de véritables placentomes géants comme ceux que nous avons observés dans une vache portant deux fœtus de $40 \mathrm{~kg}$ (poids moyen individuel d'un placentome dans le segment médian : $230 \mathrm{~g}$ ).

\section{Développement total (nombre et poids) des placentomes des différents segments de l'utérus}

Le tableau 4 indique la contribution relative des régions utérines au poids total des placentomes.

Cette répartition est la conséquence du nombre des placentomes et de leur poids relatif dans chaque zone.

Ire phénomène de compensation qui s'établit entre ces deux variables, nombre et poids individuel des placentomes, permet le maintien à peu près constant de la 
quantité de placenta disponible pour chaque fotus dans toutes les gestations examinées à un âge donné (fig. 3).

Les différences importantes de contribution des segments de la corne gravide proviennent principalement de différences de poids individuel des placentomes.

TABLEAU 4

Contribution velative (en $\mathrm{p}$. Too) des régions utérines au poids total des placentomes

\begin{tabular}{|c|c|c|c|c|c|c|c|c|c|c|}
\hline & \multirow[b]{2}{*}{ Poids du foetus } & \multirow{2}{*}{$\begin{array}{l}\text { Nbre } \\
\text { d'ani- } \\
\text { maux }\end{array}$} & \multicolumn{3}{|c|}{ Corne gravide } & \multicolumn{3}{|c|}{ Corne non gravide $\left({ }^{1}\right)$} & \multirow{2}{*}{$\begin{array}{l}\text { Corne } \\
\text { gravide }\end{array}$} & \multirow{2}{*}{$\begin{array}{l}\text { Corne non } \\
\text { gravide (1) }\end{array}$} \\
\hline & & & $\begin{array}{l}1 / 3 \\
\text { sup. }\end{array}$ & $\begin{array}{l}1 / 3 \\
\text { moy. }\end{array}$ & $\begin{array}{l}1 / 3 \\
\text { inf. }\end{array}$ & $\begin{array}{l}1 / 3 \\
\text { inf. }\end{array}$ & $\begin{array}{c}1 / 3 \\
\text { moy. }\end{array}$ & $\begin{array}{l}1 / 3 \\
\text { sup. }\end{array}$ & & \\
\hline 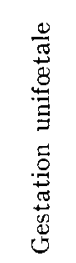 & $\begin{array}{rrr}30- & 100 \\
101- & 300 \\
301-1 & 000 \\
1001-3 & 000 \\
3001-6000 \\
6001-9000 \\
9001-50000 \\
\text { moyennes }\end{array}$ & $\begin{array}{l}7 \\
5 \\
7 \\
6 \\
6 \\
7 \\
6\end{array}$ & $\begin{array}{l}22,5 \\
18,5 \\
20,8 \\
27,2 \\
25,1 \\
29,7 \\
28,3 \\
24,6\end{array}$ & $\begin{array}{l}58,9 \\
54,0 \\
47,1 \\
40,5 \\
43,3 \\
36,2 \\
43,1 \\
46,2\end{array}$ & $\begin{array}{l}16,3 \\
23,3 \\
22,8 \\
22,0 \\
23,3 \\
16,7 \\
21,8 \\
20,9\end{array}$ & $\begin{array}{l}1,8 \\
2,6 \\
6,0 \\
5,3 \\
4,5 \\
6,1 \\
3,1 \\
4,2\end{array}$ & $\begin{array}{l}0,5 \\
1,5 \\
2,6 \\
3,6 \\
2,8 \\
8,5 \\
2,1 \\
3,1\end{array}$ & $\begin{array}{r}0,07 \\
0,05 \\
0,7 \\
1,3 \\
0,8 \\
2,4 \\
1,4 \\
1,0\end{array}$ & $\begin{array}{l}97,7 \\
95,8 \\
90,7 \\
89,7 \\
92,0 \\
82,6 \\
93,2 \\
91,7\end{array}$ & $\begin{array}{r}2,1 \\
4,2 \\
9,3 \\
10,2 \\
8,1 \\
17,3 \\
11,8 \\
8,3\end{array}$ \\
\hline 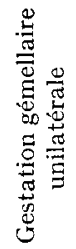 & 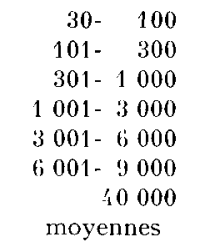 & $\begin{array}{l}1 \\
2 \\
3 \\
3 \\
2\end{array}$ & $\begin{array}{l}29,5 \\
27,2 \\
22,8 \\
26,5 \\
26,1 \\
39,3 \\
28,6\end{array}$ & $\begin{array}{l}35,7 \\
51,6 \\
48,0 \\
49,0 \\
35,2 \\
24,2 \\
40,6\end{array}$ & $\begin{array}{l}28,4 \\
16,1 \\
23,3 \\
17,5 \\
30,9 \\
20,3 \\
22,8\end{array}$ & $\begin{array}{l}3,5 \\
3,2 \\
4,0 \\
3,3 \\
3,4 \\
\\
5,4 \\
3,8\end{array}$ & $\begin{array}{l}2,3 \\
1,2 \\
1,7 \\
3,0 \\
2,7 \\
\\
6,4 \\
2,8\end{array}$ & $\begin{array}{l}0,6 \\
0,7 \\
0,2 \\
0,7 \\
1,7 \\
4,5 \\
1,4\end{array}$ & $\begin{array}{l}93,6 \\
9 ', 9 \\
9 ;, 1 \\
93,0 \\
92,2 \\
83,8 \\
91,9\end{array}$ & $\begin{array}{r}6,4 \\
5,1 \\
5,9 \\
7,0 \\
7,8 \\
16,3 \\
8,1\end{array}$ \\
\hline 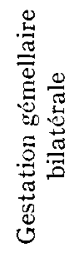 & $\begin{array}{rr}30- & 100 \\
101- & 300 \\
301-1000 \\
1001-3000 \\
3001-6000 \\
6001-9000 \\
15000 \\
\text { moyennes }\end{array}$ & $\begin{array}{l}3 \\
6 \\
8 \\
5\end{array}$ & $\begin{array}{l}12,8 \\
16,0 \\
15,0 \\
13,0\end{array}$ & $\begin{array}{l}31,5 \\
24,7 \\
23,8 \\
25,8\end{array}$ & $\begin{array}{l}11,3 \\
10,1 \\
10,8 \\
11,2\end{array}$ & $\begin{array}{r}9,{ }^{\prime} \\
10,8 \\
8,,^{\prime} \\
8,9\end{array}$ & $\begin{array}{l}23,8 \\
22,8 \\
23,0 \\
28,6\end{array}$ & $\begin{array}{l}11,2 \\
15,6 \\
19,0 \\
12,5\end{array}$ & $\begin{array}{l}55,6 \\
50,8 \\
49,6 \\
50,0\end{array}$ & $\begin{array}{l}42,4 \\
49,2 \\
50,4 \\
50,0\end{array}$ \\
\hline
\end{tabular}

(1) Ou corne contenant le foetus le moins lourd en cas de gestation gémellaire bilatérale.

La chute que l'on constate dans la contribution placentaire en passant de la corne gravide à la corne non gravide est le résultat de la diminution du nombre de placentomes dans la corne non gravide conjuguée avec la chute de leur poids individuel.

La comparaison du poids relatif de placentomes (tab1. 4) dans les deux cornes montre mieux que la comparaison de leur nombre (tabl. 2) la faible contribution de la corne non gravide à l'élaboration du tissu placentaire. 


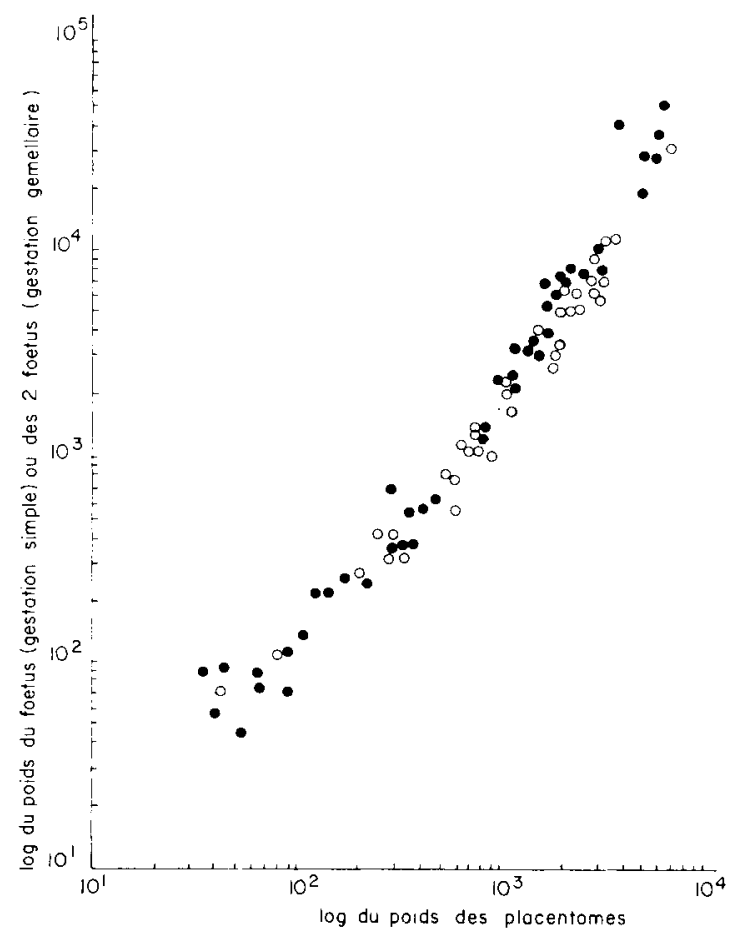

FIG. 3. - Représentation logarithmique du poids total de placenta en fonction du poids total de fotus

- Gestation simple o Gestation gémellaire

\section{Placentomes complémentaires}

Ce sont les formations placentaires constituées par l'attachement du chorion à l'utérus dans la zone intercotylédonnaire (HAMmoND, I927). Ils se forment vers 4 mois, parfois plus tôt, fréquemment vers 5 -6 mois et peuvent ne pas exister en fin de gestation.

Nous avons observé ces formations plus fréquemment dans les parties inférieure et supérieure de la corne gravide sur le corps utérin et le cervix et à la base de la corne non gravide. Leur poids atteint rarement ro p. roo du poids total des placentomes ; leur apparition ne semble pas liée à une déficience pondérale des placentomes vrais.

\section{DISCUSSION}

La vitesse de développement individuel des placentomes au cours de la gestation est étroitement liée à leur position par rapport à la zone occupée par le fœtus (gradient fœtodistal de poids individuel des placentomes). Dans la corne gravide, cette vitesse est la même en cas de gestation simple ou gemellaire bilatérale; elle est pratiquement doublée en cas de gestation gémellaire unilatérale.

L'utilisation d'un nombre maximal de caroncules n'a lieu que dans le cas des 
gestations gémellaires bilatérales. Chaque corne porte alors un nombre équivalent de placentomes et il est vraisemblable que, dans ce cas, la presque totalité des caroncules est transformée en cotylédons.

AlEXXNDER (I964) trouve, chez la Brebis, une tendance à ce qu'une proportion constante de caroncules ( $70-80 \mathrm{p}$. Ioo) devienne des cotylédons et indique qu'en gestation gémellaire chaque fœtus est attaché à un nombre plus petit de cotylédons, ces cotylédons étant 30 p. Ioo plus lourds qu'en cas de gestation simple. Nous avons vu, pour la Vache, que suivant qu'il s'agit de gestation gémellaire unilatérale ou bilatérale, chaque fotus dispose de $45 \mathrm{p}$. Ioo et $65 \mathrm{p}$. Ioo respectivement de l'effectif cotylédonnaire dont il profiterait s'il était seul. Chez la Vache comme chez la Brebis, on assiste à une croissance individuelle compensatrice des cotylédons qui permet de maintenir à peu près constant le poids de placenta par foetus dans tous les types de gestation.

Le même auteur démontre, par ablation d'un nombre défini de caroncules, que le maintien de la gestation n'est pas lié au nombre de ces caroncules : c'est seulement quand il supprime au moins 60 caroncules (sur I50) que la gestation se termine prématurément.

En ce qui concerne les cotylédons complémentaires, Kantorova (I957) pense qu'ils se forment plus tôt et en plus grand nombre dans le cas où les cotylédons principaux sont petits et en nombre plus restreint; nous n'avons pu établir le même rapport.

La valeur de supplémentation placentaire des cotylédons complémentaires est douteuse puisque, d'après le même auteur, leur structure villositaire simple ne présente pas les ramifications secondaires, favorables aux échanges, qui caractérisent les placentomes.

Quels sont les facteurs qui contrôlent le développement cotylédonnaire? HAMMoND (I927-I957) panse que le développement des placentomes est stimulé par le contact entre les membranes fotales et les surfaces caronculaires; le tissu foetal forme des doigts qui s'introduisent dans le tissu maternel, celui-ci répond par une croissance du conjonctif. C'est l'implantation qui a lieu entre le $3^{\mathrm{e}}$ et le $35^{\mathrm{e}}$ jour de gestation (Melton, Berry, Butrar, I95I; Chang, I952). Si la distension exercée par l'embryon et les liquides est la première condition d'implantation, on conçoit le développement dégressif du placenta à mesure qu'on s'éloigne de la région fotale et, particulièrement, la chute observée lors du passage dans la corne non gravide due peut-être à la constriction au niveau du corps utérin.

Cet effet de distension expliquerait l'effectif des placentomes suivant les zones, mais le développement pondéral des cotylédons peut répondre en même temps à une régulation hémodynamique ou endocrinienne. Ainsi ALEXANDER et WILLIAMS (I966) montrent chez la Brebis que la progestérone stimule l'invasion du cotylédon maternel par les villosités fœtales, le cotylédon maternel répondant peu à cette invasion. Ils obtiennent, par supplémentation de progestérone à des doses élevées, des placentomes comparables à ceux des bovins, le cotylédon maternel étant enfermé dans le cotylédon fœetal, contrairement aux placentomes ovins.

Il apparaît nettement dans nos résultats que si on compare la contribution des cornes utérines à la formation du placenta, on ne peut opposer gestation simple et gestation gémellaire, mais gestation unilatérale et gestation bilatérale. En effet, en gestation unilatérale (simple ou gémellaire), la corne non gravide, avec $30 \mathrm{p}$. Ioo des 
placentomes participe pour moins de Io p. Ioo à la formation du placenta, alors qu'en gestation bilatérale, la contribution de chaque corne est équivalente. Cependant, gestation simple et gestation gémellaire se différencient quant au poids total des placentomes, chaque foetus bénéficiant, à un âge donné, d'un poids de placenta sensiblement analogue.

\section{RÉSUMÉ}

Sur 80 utérus de vaches gestantes ( 44 gestations simples, I 2 gémellaires unilatérales et 24 gémellaires bilatérales), on a étudié le nombre, la répartition et le développement pondéral des placentomes. Le terme de placentome désigne l'unité comprenant le cotylédon maternel issu du caroncule et le cotylédon foctal correspondant.

En cas de gestation unilatérale, simple ou gémellaire, le nombre moyen de placentomes est beaucoup plus élevé dans la corne gravide que dans l'autre : 68 contre 28 pour les gestations simples, 57 contre 28 pour les gestations gémellaires. En cas de gestation double bilatérale, ce nombre est identique dans les deux cornes : 64 contre 64 .

Le poids des placentomes est étroitement lié à leur distance du ou des foetus, au nombre et à la position de ces derniers. Lorsque la gestation gémellaire est unilatéralc, on trouve, dans l'aire voisine de l'implantation des fœetus, des placentomes géants, deux fois plus gros que ceux qui existent au cours de gestation simple ou gémellaire bilatérale. Lors d'une gestation double bilatérale, chaque corne contribue, pour une part égale, au développement des placentomes. Par contre, lors d'une gestation unilatérale, simple ou gémellaire, la corne non gravide y contribue pour moins de ro p. Ioo.

\section{SUMMARY}

\section{BIOMETRIC STUDY OF "PIACENTOMES " IN SINGLE OR TWIN BOVINE PREGNANCIES}

The number, distribution and weight development of "placentomes " have been studied in the uteri of 80 pregnant cows (44 single pregnancies, I 2 unilateral twin and 24 bilateral twin pregnancies). The term "placentome" designates a unit comprising the maternal cotyledon, issue of the caroncule, and the corresponding fœtal cotyledon.

In the case of unilateral single or twin pregnancies, the average number of "placentomes" is much higher in the pregnant horn than in the other one ; that is, 68 against 28 for single pregnancies, 57 against 28 for twin pregnancy. For bilateral double pregnancy, this number is similar in both horns; that is, $6_{4}$ against $6_{4}$.

The weight of the "placentomes" is closely related to the distance separating them from the foetus, and to the number and position of the fotuses. When the twin pregnancy is unilateral, huge " placentomes", twice as big as those which exist during single or bilateral twin pregnancy, may be scen near to the foetus implantation. In a bilateral double pregnancy, each horn contributes equally to the development of the " placentomes."

On the contrary, during a unilateral single or twin pregnancy, the contribution of the nonpregnant horn is less than ro p. 100.

\section{RÉFÉRENCES BIBIIOGRAPHIQUES}

Alexaxder G., I964. Studies on the placenta of the sheep (Ovis aries L.) placental size. J. Reprod. Fert., 7, 289-305.

Alexander G., $\mathbf{r}^{6} 6_{4}$. Studies on the placenta of the sheep (Ovis aries L.). Effect of surgical reduction in the number of caruncles. J. Reprod. Fert., 7, 307-322.

Alexander G., Williams D., I966. Progesterone and placental development in the sheep. J. Endocr., 34, $24 \mathrm{I}-246$. 
Chang M. C., r952. Development of bovine blastocyst with a note on implantation. Anat. Rec., 113, I43-I $6 \mathrm{r}$.

FourNier C., I963. Dynamique morphologique du fatus bovin. Contribution à la determination de l'âge fatal. Thèse vét., Alfort

HaFEz E. S. E., Rajakowski E., I964. Placental and fetal development during multiple bovine pregnancy. Anat. Rec., 150, 303-3I6.

Hafez E. S. E., Jainudeen M. R., Lindsay D. R., i965. Gonadotropin-induced Twinning and related phenomena in beef cattle. Acta Endocr., 50, Suppl. roz, r-44.

Hammond J., I927. Reproduction in the cow, ch. IV. Pregnancy. Cambridge, Univ. l'ress.

Hammond J., I957. Progress in the Physiology of farm animals. Vol. 3, Butterworths Sci. Publ. London.

Kantorow. V. J., I957. The structure of intercotyledonal chorion in Bos taurus (russe). Dokl. Akad. Nauk. S. S. S. R., 113, $233^{-236 .}$

Makowski E. L., Meschia G., Droegemueller W., Battaglia F. C., ig68. Distribution of uterine blood flow in the pregnant sheep. Am. J. Obst. Gynec. 101, 409-4I2.

Melton A. A., Berry R. O., Butler O. D., I95x. The interval between the time of ovulation and attachment of the bovine embryo. J. anim. Sci., 10, 993-Ioo3.

Winters L. M., Green W. W., Comstock R. E., I953. Prenatal development of the bovine. Tech. Bull. Minnesota agric. Exp. Sta., 151, I-43. 